\title{
PEGylated $\mathrm{MoS}_{2}$ Nanosheets: A Dual Functional Photocatalyst for Photodegradation of Organic Dyes and Photoreduction of Chromium from Aqueous Solution
}

\author{
Madima Ntakadzeni' ${ }^{1}$, William Wilson Anku ${ }^{*}$, Neeraj Kumar ${ }^{1}$, Penny Poomani Govender ${ }^{1}$, \\ Leelakrishna Reddy ${ }^{2}$ \\ ${ }^{1}$ Department of Applied Chemistry, University of Johannesburg, Doornfontein Campus 2028, \\ Johannesburg, South Africa \\ ${ }^{2}$ Department of Physics, University of Johannesburg, Doornfontein Campus 2028, \\ Johannesburg, South Africa
}

Received: 22 $2^{\text {nd }}$ February 2018; Revised: 24th October 2018; Accepted: 30th October 2018; Available online: 25th January 2019; Published regularly: April 2019

\begin{abstract}
This article reports the synthesis of PEGylated microspheres of $\mathrm{MoS}_{2}$ nanosheets through the hydrothermal method and its application in rhodamine B and methylene blue dyes photodegradation, and photoreduction of chromium(VI) to chromium(III) in water under illumination with visible light. The catalyst was characterized using X-ray Diffraction (XRD), Transmission Electron Microscopy (TEM), Field Emission Scanning Electron Microscopy (FESEM), Energy Dispersive X-ray Spectroscopy (EDS), Fourier Transform Infra Red (FTIR), Thermo-gravimetric Analysis (TGA), and UV-Vis spectroscopies. XRD result reveals the $\mathrm{MoS}_{2}$ nanosheets to be present in the hexagonal phase of MoS 2 . SEM, TEM, and HRTEM images show that the synthesised sample has spherical shapes made up of several thin sheets of $\mathrm{MoS}_{2}$. The catalyst showed visible light responsivity with a calculated band gap of $1.92 \mathrm{eV}$. The $\mathrm{MoS}_{2}$ nanosheets exhibited high degradation efficiency against both dyes. The RhB and MB dyes experienced degradation efficiencies of $97.30 \%(\mathrm{RhB})$ and $98.05 \%(\mathrm{MB})$ in $75 \mathrm{~min} 90 \mathrm{~min}$, respectively. The $\mathrm{MoS}_{2}$ photocatalyst is also observed to be effective in photocatalytic reduction of $\mathrm{Cr}(\mathrm{VI})$ and displayed $91.05 \%$ reduction of $\mathrm{Cr}(\mathrm{VI})$ to $\mathrm{Cr}$ (III) in $75 \mathrm{~min}$. The results reveal that the synthesised $\mathrm{MoS}_{2}$ nanosheet is a good photocatalytic material for degradation of dyes and reduction of $\mathrm{Cr}$ (VI) to $\mathrm{Cr}$ (III) in water. Copyright @ 2019 BCREC Group. All rights reserved
\end{abstract}

Keywords: Photocatalyst; Photodegradation; Photoreduction; Dyes; Chromium, $\mathrm{MoS}_{2}$

How to Cite: Ntakadzeni, M., Anku, W.W., Kumar, N., Govender, P.P., Reddy, L. (2019). PEGylated $\mathrm{MoS}_{2}$ Nanosheets: A Dual Functional Photocatalyst for Photodegradation of Organic Dyes and Photoreduction of Chromium from Aqueous Solution. Bulletin of Chemical Reaction Engineering \& Catalysis, 14 (1): 142-152 (doi:10.9767/bcrec.14.1.2258.142-152)

Permalink/DOI: https://doi.org/10.9767/bcrec.14.1.2258.142-152

\section{Introduction}

An increase in the world population together with the proliferation of factories and industrial

\footnotetext{
* Corresponding Author.

E-mail: williamanku85@gmail.com (W.W. Anku); krishr@uj.ac.za (L. Reddy)
}

development has led to increased energy crisis and environmental contamination [1]. Environmental contaminations issues are mostly associated with hazardous wastes, and toxic organic pollutants discharged into water bodies. Most of the coloured effluents emanating from industries, particularly the textile industries, contain 
toxic organic dyes and dyestuffs [2-4].

Heavy metals such as chromium (Cr) which is usually used in various applications including its use in alloys, steel manufacturing, chrome plating, leather tanning and wood processing $[5,6]$ exists in the environment as a result of direct discharge from industries. It is regarded as a harmful entity owing to its highly toxic nature. Chromium exists in two different forms, i.e. $\mathrm{Cr}(\mathrm{VI})$ and $\mathrm{Cr}(\mathrm{III})$ with $\mathrm{Cr}(\mathrm{VI})$, being the most harmful form present in the aquatic environment. Dyes and $\mathrm{Cr}(\mathrm{VI})$ contaminated wastewater discharged from industries pollute water resources and pose life-threatening health effects to humans and wildlife [7]. As a result, issues relating to water pollution with heavy metals and organic dyes are receiving greater attention with a focus on the removal of dye molecules and $\mathrm{Cr}(\mathrm{VI})$ from wastewater before their final discharge into the environment.

Many efforts have been put into the removal of organic pollutants from wastewater due to the threat they pose to all forms of life. Recently, various techniques have been applied for the elimination of heavy metals and organic dyes from polluted water which includes chemical oxidation [8], extraction [9], adsorption [10], photocatalytic activity [11], and biochemical method [12]. Amongst them, photocatalytic degradation and photocatalytic reduction of organic pollutants and heavy metals using nanostructured photocatalyst semiconductor materials have been considered to be the most promising and sustainable strategies for water decontamination and environment remediation [1315]due to their simplicity of design, low cost, flexibility and eco-friendliness [16,17]. Photocatalytic degradation of organic pollutants is a process that combines catalysis with solar radiation $[18,19]$ to degrade the dyes into harmless compounds $\left(\mathrm{CO}_{2}\right.$ and $\left.\mathrm{H}_{2} \mathrm{O}\right)$ and also to reduce $\mathrm{Cr}(\mathrm{VI})$ into harmless $\mathrm{Cr}$ (III). In this regard, a photocatalytic semiconductor material absorbs light of energy more than or equal to its band-gap, resulting in electrons and holes generations followed by the formation of free radicals that are responsible for the oxidation of the contaminants.

Amongst the known photocatalyst, titanium dioxide $\left(\mathrm{TiO}_{2}\right)$ is the broadly used photocatalyst of choice due to the fact that it possesses certain peculiar features including strong oxidizing power, chemical stability, nontoxicity and low cost [20,21]. Irrespective of the various advantages, the use of $\mathrm{TiO}_{2}$ and other photocatalysts in industrial scale photocatalysis is still restricted due to certain inherent shortfalls of these photocatalysts. These shortfalls include rapid recombination of excited charge carriers, poor chemical stability and wide band gap energies which only permits the catalysts to use ultraviolet (UV) light instead of the more abundant visible light. The UV light represents only about $4 \%$ of the solar spectrum while the visible light accounts for about $45 \%$ of the spectrum [22]. Therefore, it has become necessary to fabricate photocatalysts that are capable of utilizing visible light with efficient charge carriers separation abilities [23,24].

Molybdenum disulfide $\left(\mathrm{MoS}_{2}\right)$ is a low-cost, non-toxic and abundant semiconductor material with a small direct energy band gap (1.8 $\mathrm{eV})$ in nanoscale and indirect energy band gap $(1.2 \mathrm{eV})[25,26]$ in bulk form. It has been extensively used in electronics, energy conversion, and storage devices, but the potential of $\mathrm{MoS}_{2}$ nanostructures in water purification and metal reduction has not been fully explored. In this study, we employed the one-step hydrothermal synthesis method to synthesize microspheres of $\mathrm{MoS}_{2}$ nanosheets using polyethylene glycol (PEG) as a capping agent. The photocatalytic activity of the synthesized photocatalyst was explored for the photodegradation of Rhodamine (RhB), Methyl blue (MB) and photoreduction of $\mathrm{Cr}(\mathrm{VI})$ to $\mathrm{Cr}(\mathrm{III})$ in aqueous solution.

\section{Materials and Method}

\subsection{Materials}

Ammonium hexamolybdate tetrahydrate (AHM), polyethylene glycol (PEG, average $\mathrm{M}_{\mathrm{n}}$ 400), thiourea, ethanol (99.8\%), methylene blue, rhodamine $\mathrm{B}$, and potassium dichromate $\left(\mathrm{K}_{2} \mathrm{Cr}_{2} \mathrm{O}_{7}\right)$ were all obtained from SigmaAldrich and used without further purifications. Standard solution of the methylene blue and rhodamine B dyes were prepared through the dissolution of the appropriate masses of the dyes in deionized water $(1000 \mathrm{~mL})$. The desired dye concentrations $(20 \mathrm{mg} / \mathrm{L})$ were obtained through dilution of the prepared standard solutions. The $\mathrm{Cr}(\mathrm{VI})$ solution (10 ppm) was prepared by dissolving the calculated amount of $\mathrm{K}_{2} \mathrm{Cr}_{2} \mathrm{O}_{7}$ in deionized water $(1000 \mathrm{~mL})$.

\subsection{Synthesis of $\mathrm{MoS}_{2}$ Nanosheets using PEG as a Capping Agent}

The microsphere $\mathrm{MoS}_{2}$ nanosheets were synthesised in a Teflon-lined autoclave via a hydrothermal route by using AHM and thiourea as the sources of molybdenum and sulphur, respectively. PEG-400 was used as a capping agent. The procedure of the preparation was as follows: A $1.200 \mathrm{~g}$ of AHM was dissolved 
in $15 \mathrm{~mL}$ distilled water by a magnetic stirrer for $15 \mathrm{~min}$. In another vial, $1.00 \mathrm{~g}$ of PEG-400 was also dissolved distilled water $(15 \mathrm{~mL})$. The PEG-400 solution was then introduced into the vial containing the AHM solution after which the mixture was stirred for $30 \mathrm{~min}$. Thereafter, $2.400 \mathrm{~g}$ of thiourea was added the above mixture and the resultant mixture was stirred for another $20 \mathrm{~min}$. The final solution was then transferred into $50 \mathrm{~mL}$ Teflon lined autoclave and reacted at $220{ }^{\circ} \mathrm{C}$ for $24 \mathrm{~h}$. A black precipitate was obtained at the end of the reaction and was separated by centrifuging the suspension for $20 \mathrm{~min}(7830 \mathrm{rpm})$. The Black $\mathrm{MoS}_{2}$ nanosheet was collected and washed several times with distilled water and ethanol. It was then dried in an oven overnight at $60{ }^{\circ} \mathrm{C}$. A schematic diagram for the synthesis of the $\mathrm{Pe}$ gylated microsphere $\mathrm{MoS}_{2}$ nanosheets is presented in Scheme 1.

\subsection{Materials Characterizations}

The crystal structure of the product was investigated by X-ray diffraction (XRD) with $\mathrm{Cu}$ Ka radiation $(\lambda=0.15418 \mathrm{~nm})$. The morphology and structure of the sample were characterized by transmission electron microscopy (TEM) and field emission scanning electron microscopy (FESEM) equipped with energy dispersive Xray spectroscopy (EDS). Fourier transformed infrared (FTIR) spectrometer was used to de- tect the functional groups in the sample. Thermo-gravimetric analysis (TGA) was also performed to determine the thermal stability of PEGylated $\mathrm{MoS}_{2}$ nanosheets. UV-Vis spectrometer was used for the determination of the energy band gap and also for the evaluation of photocatalytic activities.

\subsection{Photocatalytic Experiment}

\subsubsection{Photodegradation of dye molecules}

Photodegradation properties of the microsphere $\mathrm{MoS}_{2}$ nanosheets were assessed by monitoring its ability to degrade $\mathrm{RhB}$ and $\mathrm{MB}$ dyes in aqueous solutions. In both experiments, $50 \mathrm{mg}$ of the photocatalyst was dispersed in $100 \mathrm{~mL}$ (10 ppm) each of $\mathrm{RhB}$ and $\mathrm{MB}$ dye aqueous solutions. Prior to irradiation, the dye solutions with suspended photocatalysts in a round bottom flask were stirred in dark conditions to ensure that the surfaces of the catalysts were saturated with the dyes. The solutions were then placed in a photoreactor and were exposed to visible light under magnetic stirring conditions. The visible light was produced by attaching a UV filter to a solar simulator $(250 \mathrm{~W})$ which was the source of light for the experiment. At a regular time interval of $15 \mathrm{~min}, 4 \mathrm{~mL}$ of irradiated solutions of each dye was collected and centrifuged to remove the catalysts, and the supernatants were used for the evaluation of photodegradation. The su-

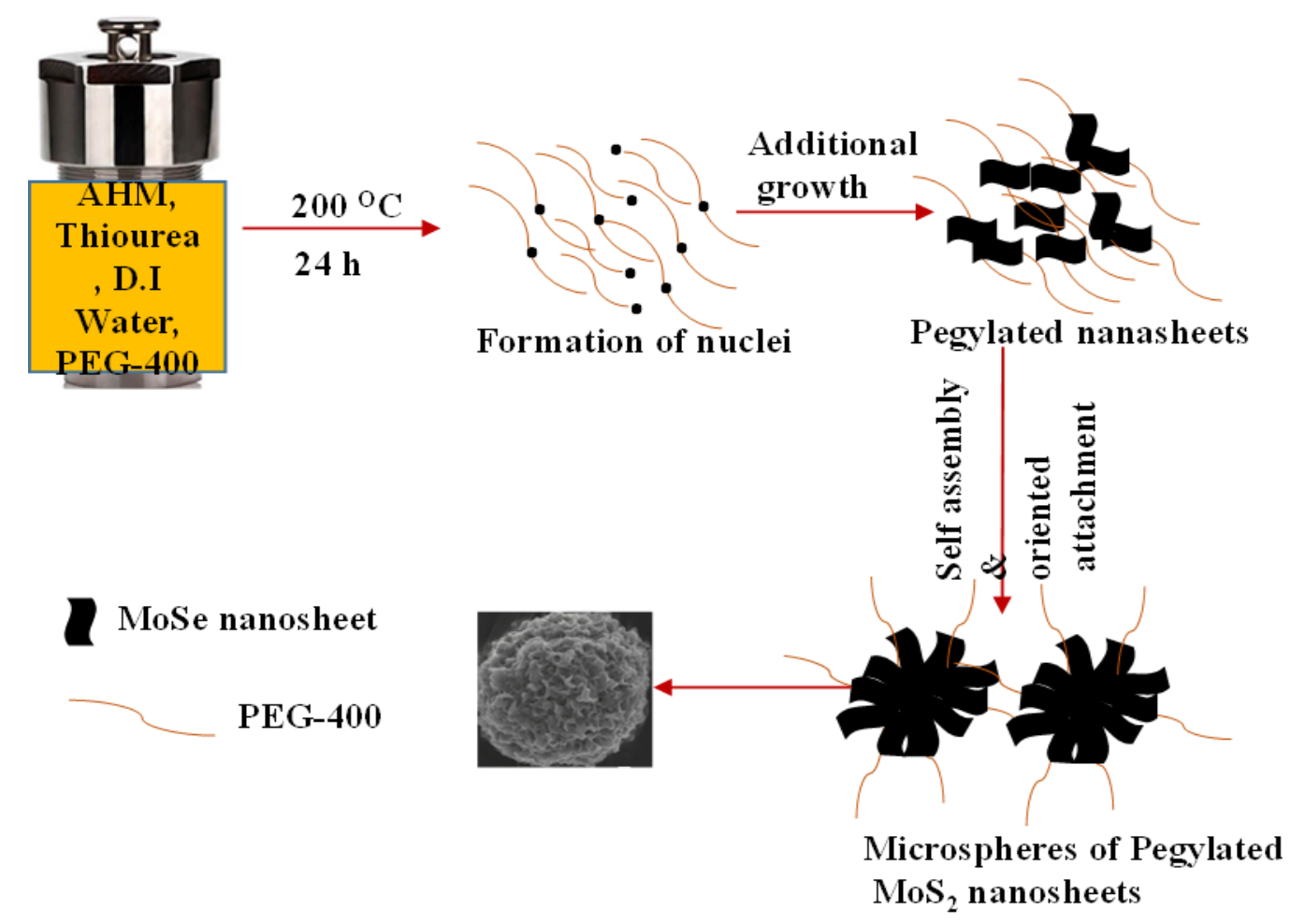

Scheme 1. A schematic diagram for the synthesis of the Pegylated microsphere $\mathrm{MoS}_{2}$ nanosheets 
pernatants were analysed using UV-Vis spectrophotometer to record the intensity of the absorption peaks of $\mathrm{RhB}(555 \mathrm{~nm})$ and $\mathrm{MB}(665$ $\mathrm{nm})$.

\subsubsection{Photoreduction of Chromium Cr(VI)}

The photocatalytic performance of the synthesised catalyst was also evaluated through photocatalytic degradation of $\mathrm{Cr}(\mathrm{VI})$ under visible light irradiation. The photocatalyst (50 $\mathrm{mg}$ ) was suspended in $\mathrm{Cr}(\mathrm{VI})$ solution $(100 \mathrm{~mL}$, $10 \mathrm{ppm}$ ) and the mixture was stirred for $20 \mathrm{~min}$ under a dark condition so as to attain adsorption-desorption equilibrium. Then, the suspension was illuminated with visible light for 75 minutes and at every 15 minutes intervals, 4 $\mathrm{mL}$ of the suspension was withdrawn and centrifuged to separate the photocatalyst and the $\mathrm{Cr}(\mathrm{VI})$. The process of photocatalytic reduction was monitored using UV-Vis spectrophotometer to record the intensity of the absorption peaks of $\mathrm{Cr}(\mathrm{VI})$ at $350 \mathrm{~nm}$.

\subsubsection{Catalyst recyclability study}

The recyclability of the $\mathrm{MoS}_{2}$ nanosheets for the degradation of $\mathrm{RhB}$ and $\mathrm{MB}$ dyes and re- duction of $\mathrm{Cr}(\mathrm{VI})$ to $\mathrm{Cr}$ (III) was also performed by adhering to the procedure described in section 2.4.1 and 2.4.2, respectively. The reusability assessments were executed 5 successive times. At the end of each degradation cycle, the catalysts were separated from the solution by centrifugation. They were washed, dried at 60 ${ }^{\circ} \mathrm{C}$ for $12 \mathrm{~h}$, and reused.

\section{Results and Discussions}

\subsection{Characterizations}

The morphologies of the synthesised microspheres of $\mathrm{MoS}_{2}$ nanosheets were obtained via FESEM, TEM, and HRTEM. The FESEM image of the pure $\mathrm{MoS}_{2}$ in Figure 1(a) clearly shows a cluster of $\mathrm{MoS}_{2}$ thin sheets. That of the Pegylated $\mathrm{MoS}_{2}$ (Figure 1(b)) is of microspherical shapes made up of several thin sheets of $\mathrm{MoS}_{2}$. Thus, the PEG-400 played the role of a capping agent. TEM image from Figure 1(c) shows that the sample is made up of sheets of $\mathrm{MoS}_{2}$. Figure1(d) which shows a high resolution-TEM image of the sample, reveals a layered structure of nanosheets. The distance between the adjacent layers was esti-
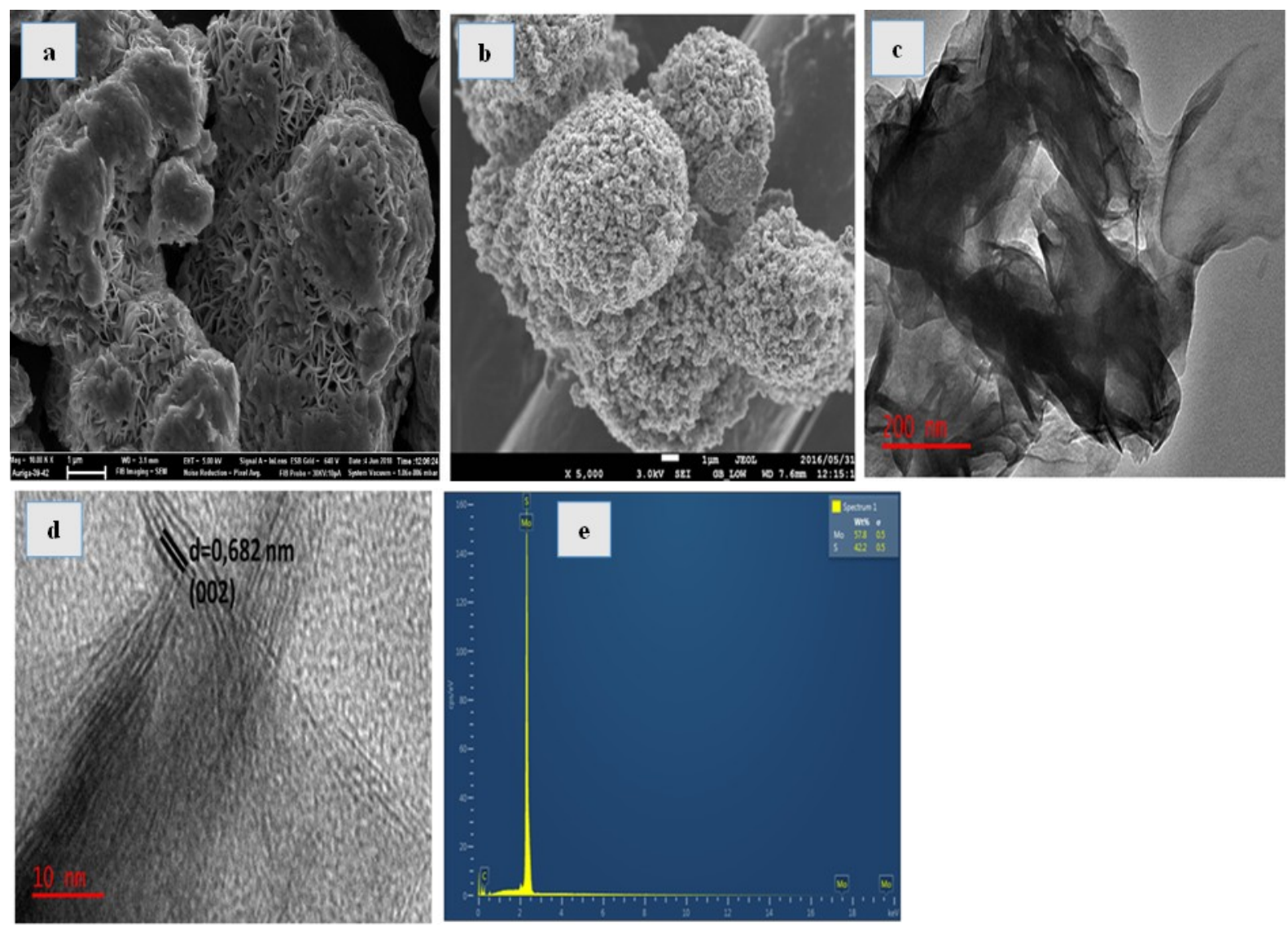

Figure 1. (a) FESEM image, (b) TEM image, (c) HRTEM image, and (d) EDX spectrum of the microspheres of $\mathrm{MoS}_{2}$ nanosheets 
mated to be $0.682 \mathrm{~nm}$ corresponding to $(022)$ planes of hexagonal $\mathrm{MoS}_{2}$ nanosheets. For the identification of elemental composition in the sample, SEM equipped with EDS was used. A typical EDS spectrum of $\mathrm{MoS}_{2}$ shown in Figure 1(e) reveals the presence of Mo and S. The elemental C and O, come from the PEG-400 used as capping agents during the synthesis.

Figure 2 reveals the XRD pattern of the synthesized $\mathrm{MoS}_{2}$ nanosheets. All the diffraction peaks are indexed as hexagonal $\mathrm{MoS}_{2}$ (ICDD: $00-037-1492)$. The diffraction peak at $14.28^{\circ}$ corresponds to (002) planes with a $d$-spacing of $0.620 \mathrm{~nm}$ which was calculated from Bragg's equation, whereas the $\mathrm{d}$-spacing of pristine $\mathrm{MoS}_{2}$, according to ICDD: 00-037-1492, is 0.612 $\mathrm{nm}$ indicating enlarged interlayer spacing in the nanosheets.

The peak at $33.96^{\circ}$ correspond to (100) planes and indicates reduced spacing of 0.264 $\mathrm{nm}$ compared with $0.274 \mathrm{~nm}$ of pristine $\mathrm{MoS}_{2}$. The two peaks at $40.27^{\circ}$ and $60.28^{\circ}$ correspond to (103) and (110) planes with $d$-spacing of $0.224 \mathrm{~nm}$ and $0.153 \mathrm{~nm}$, respectively. The results prove that the PEGylated $\mathrm{MoS}_{2}$ nanosheets were successfully prepared. The peaks were found to be broad which indicate

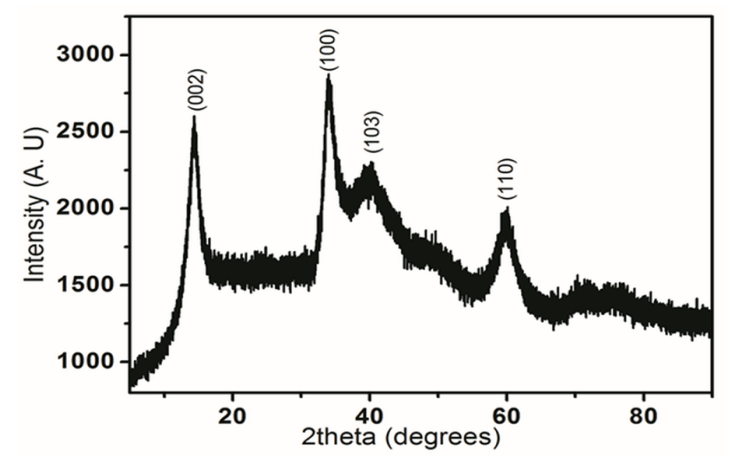

Figure 2. XRD pattern of the microspheres of $\mathrm{MoS}_{2}$ nanosheets

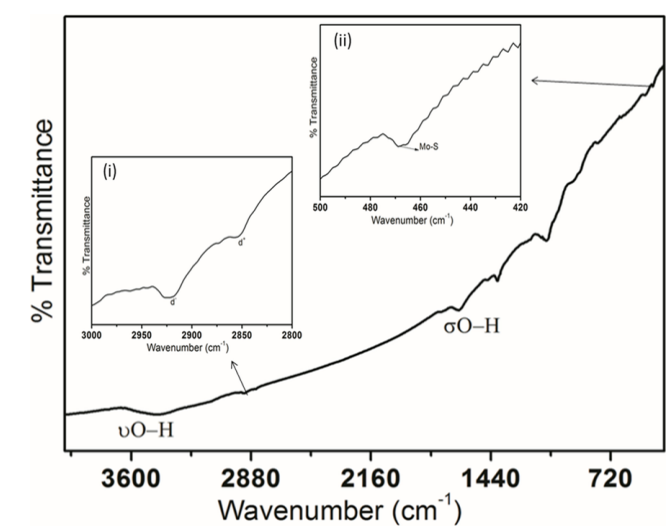

Figure 3. FTIR spectrum of the microspheres of $\mathrm{MoS}_{2}$ nanosheets smaller crystallite.

Figure 3 illustrates the FTIR spectrum of the photocatalyst. Two vibrational modes at about 3435 and $1635 \mathrm{~cm}^{-1}$ are observed and assigned to the hydroxyl functionalities of adsorbed moisture from the atmosphere on the $\mathrm{MoS}_{2}$ nanosheets. Figure 3(i), which is the inserted spectrum from $2800-3000 \mathrm{~cm}^{-1}$ range, shows the methyl symmetric and asymmetric stretches which are attributed to adsorbed PEG molecules on the $\mathrm{MoS}_{2}$ nanosheets. The inserted spectrum of Figure 3(ii) which ranges from $400-500 \mathrm{~cm}^{-1}$, exhibits a characteristic vibrational mode at $455 \mathrm{~cm}^{-1}$ and was assigned to the Mo-S linkage of the synthesised $\mathrm{MoS}_{2}$ sample.

To assess the thermal stability of the prepared PEGylated $\mathrm{MoS}_{2}$ nanosheets, TGA analysis of the sample was executed in the temperature range of 25 to $800{ }^{\circ} \mathrm{C}$ in air flow. The result is shown in Figure 4 . The result shows four stages of weight lost. The first weight loss from 25 to $130{ }^{\circ} \mathrm{C}$ happens immediately after heating begins. This weight loss is assigned to the evaporation of absorbed water/moisture from the atmosphere.

The second stage of weight loss occurs from 245 to $340{ }^{\circ} \mathrm{C}$ and it is attributable to the decomposition of adsorbed polyethylene glycol molecules on the $\mathrm{MoS}_{2}$ surfaces [32]. The third weight loss which occurred from 355 to $456{ }^{\circ} \mathrm{C}$ is assigned to the oxidation of $\mathrm{MoS}_{2}$ to hexagonal- $\mathrm{MoO}_{3}\left(\mathrm{~h}-\mathrm{MoO}_{3}\right)$ and finally phase transformation to $\mathrm{a}-\mathrm{MoO}_{3}$. The last sharp stage of weight lost transpires possibly because of the decomposition of a- $\mathrm{MoO}_{3}$.

UV-Vis spectrum (Figure 5 (a)) was obtained in order to probe the optical properties of the synthesized sample. The $\mathrm{MoS}_{2}$ nanosheet demonstrates the ability to absorb light in the wavelength range of 200 to $800 \mathrm{~nm}$. This indicates the ability of the catalyst to absorb light

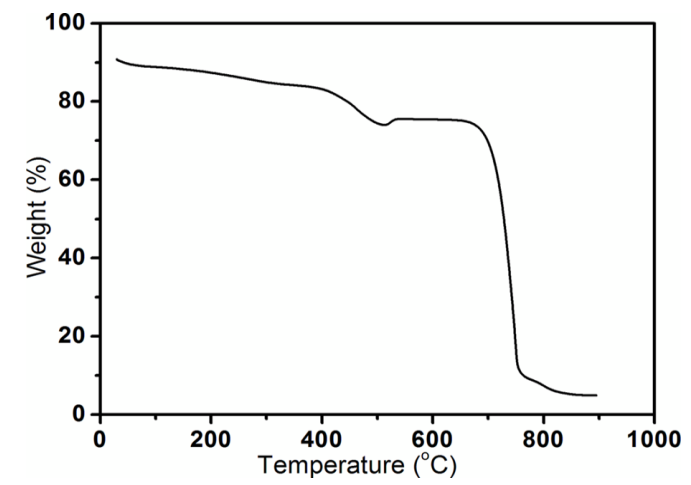

Figure 4. TGA curve of the microspheres of $\mathrm{MoS}_{2}$ nanosheets 
in both UV and visible light range of the electromagnetic spectrum.

The energy band of prepared $\mathrm{MoS}_{2}$ nanosheets was calculated using equation (1):

$$
\alpha h v=A\left(h v-E_{g}\right)^{n}
$$

where $a, v, A$, and $E_{g}$ are the absorption coefficient, light frequency, proportionality constant and band gap energy, respectively. $n$ describes the type of transition in the semiconductor.

$\mathrm{MoS}_{2}$ exhibits a direct transition in nanoscale and indirect transition in bulk form, hence the value of $n$ was taken as $1 / 2$ for the synthesised $\mathrm{MoS}_{2}$ nanosheets. Figure 5(b) shows the energy band gap of the catalyst which was estimated from the plot of $(a h v)^{2}$ versus $h v$ by extrapolating the straight line to $\mathrm{X}$-axis intercept. The result indicates that the synthesised $\mathrm{MoS}_{2}$ nanosheet has the energy band gap of $1.92 \mathrm{eV}$. This band gap value means that the $\mathrm{MoS}_{2}$ nanosheet is visible light active and hence its ability to absorb light in the visible range (Figure 5(a)).

\subsection{Photocatalytic Studies}

3.2.1 Photocatalytic degradation of RhodamineB

Figure 6(a) illustrations the changes in the absorption spectrum of $\mathrm{RhB}$ dye with respect to irradiation time over the synthesised $\mathrm{MoS}_{2}$ nanosheets. The typical absorption peak of $\mathrm{RhB}$ which occurs at $555 \mathrm{~nm}$ is noted to decreases progressively with an increase in irradiation time. This reduction in the peak intensity indicates the gradual degradation of the dye with increasing time. Figure 6(b) displays the photocatalytic degradation rate of $\mathrm{RhB}$ as a function of time with light and without irradiation with light. The concentration of RhB was observed to reduce progressively as the irradiation time increases.

The results also show that the rate of degradation of RhB by $\mathrm{MoS}_{2}$ nanosheets in the presence of light is faster as compared to the degradation rate in the absence of light, which implies that light plays an important role in the (a)

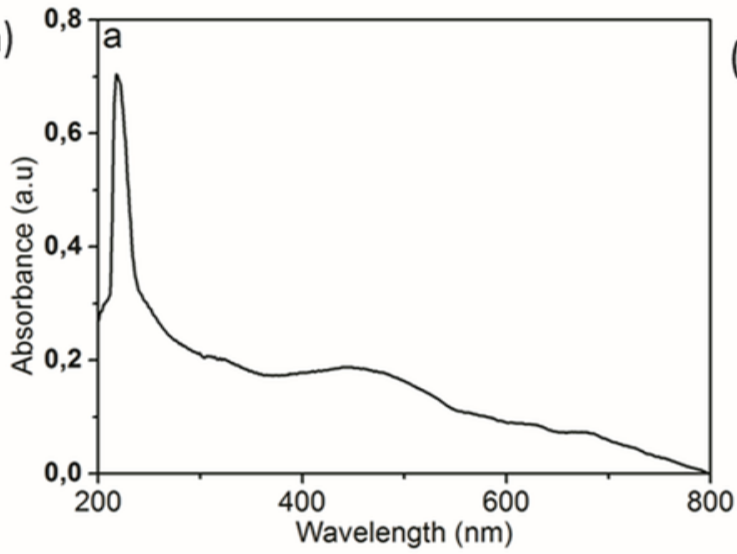

(b)

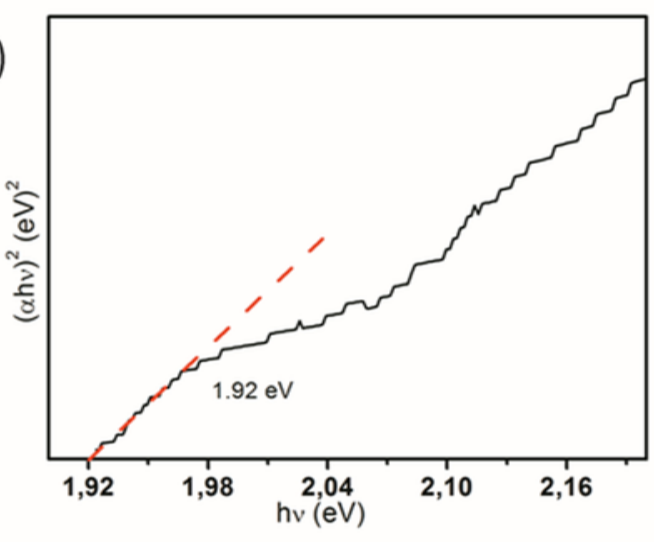

Figure 5. (a) UV-Vis and (b) Tauc plot estimated band-gap energy of the microspheres of $\mathrm{MoS}_{2}$ nanosheets
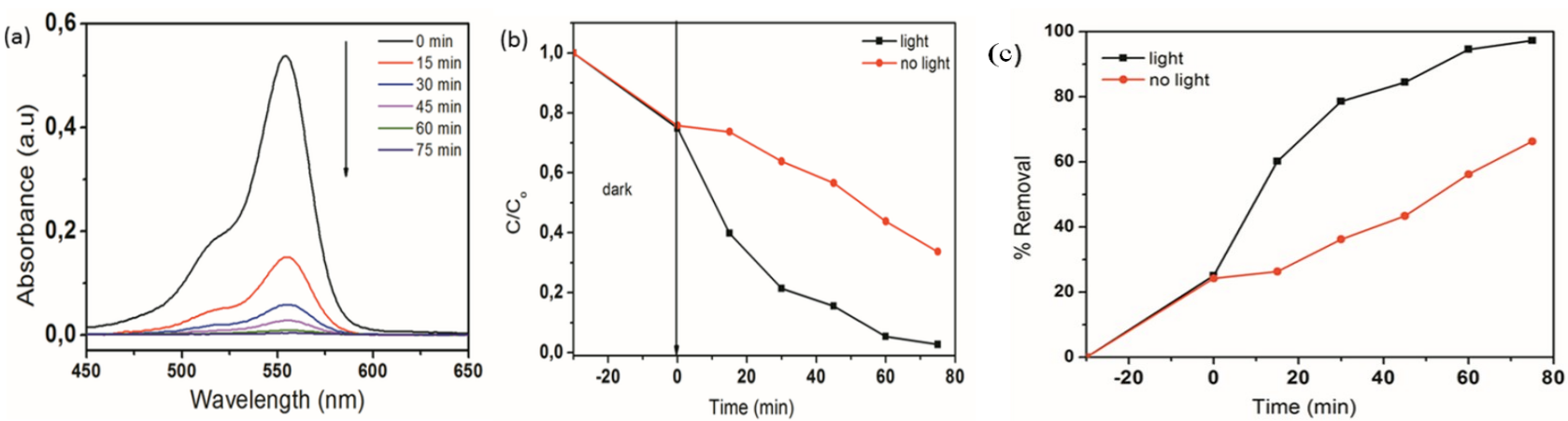

Figure 6. (a) UV-Vis spectroscopic changes of $\mathrm{RhB}$ aqueous solution in the presence $\mathrm{MoS}_{2}$ nanosheets, (b) Photocatalytic degradation rate of $\mathrm{RhB}$ as a function of time and (c) Percent removal of RhB in the presence of $\mathrm{MoS}_{2}$ nanosheets as a function of irradiation time 
photodegradation of $\mathrm{RhB}$. The reduction in the concentration of the dye in the dark can be attributed to adsorption of its molecules onto the surface of the catalyst which usually takes place prior to degradation.

The following equation was used to determine the \% removal of $\mathrm{RhB}$ at a particular time $t$ :

$$
\% \text { Removal }=\left(1-\frac{c}{c_{o}}\right) \times 100
$$

It was observed that the prepared $\mathrm{MoS}_{2}$ nanosheets showed greater efficiency in the degradation of $\mathrm{RhB}$ under light irradiation than in the absence of light, since the percentage removal in the presence of light was obtained to be $97.3 \%$ and only $66.3 \%$ was achieved in the absence of light as shown in Figure 6(c).

\subsubsection{Photocatalytic degradation of Methyl Blue}

The variation in the absorption spectrum of MB (10 ppm) dye with respect to irradiation time over the synthesised $\mathrm{MoS}_{2}$ photocatalyst is shown in Figure 7(a). The characteristic absorption peak at $665 \mathrm{~nm}$ decreases with an increase in irradiation time. The photodegradation rate of MB is shown in Figure 7(b). The results show that the concentration of MB decreases as the irradiation time increases indicating a gradual degradation of the dye with increasing time. To determine removal efficiency of $\mathrm{MB}$ by $\mathrm{MoS}_{2}$ nanosheets, the equation (2) was used. As evident from Figure 7(c), the prepared $\mathrm{MoS}_{2}$ photocatalyst degrades $98.05 \%$ of MB in 90 min. Thus the $\mathrm{MoS}_{2}$ nanosheet exhibit high degradation efficiency against both $\mathrm{RhB}$ and MB dyes.

\subsubsection{Chromium photoreduction studies}

The photoreduction study was undertaken to evaluate the capability of $\mathrm{MoS}_{2}$ nanosheets to photoreduce $\mathrm{Cr}$ (VI) to $\mathrm{Cr}$ (III). The UV-Vis absorption spectra of $\mathrm{Cr}(\mathrm{VI})$ reduction is presented in Figure 8. The figure shows a consistent decrease in the absorption spectrum of $\mathrm{Cr}(\mathrm{VI})$ with time in the presence of $\mathrm{MoS}_{2}$ nanosheets which suggests that the $\mathrm{Cr}(\mathrm{VI})$ was gradually being converted to $\mathrm{Cr}$ (III) by the catalyst with increasing irradiation time. Figure 8(b) indicates the rate of $\mathrm{Cr}(\mathrm{VI})$ conversion as a function of time, and it was observed that the concentration of $\mathrm{Cr}(\mathrm{VI})$ decreases as the irradiation time increases. These observations mean the $\mathrm{Cr}(\mathrm{VI})$ become progressively converted to $\mathrm{Cr}$ (III) with increasing time. In order to investigate the role of kinetics on the photocatalytic reduction of $\mathrm{Cr}(\mathrm{VI})$, the plot of $-\ln \left(C / C_{o}\right)$ versus time $(t)$ was obtained and the result is shown in Figure 8 (c). The plot was fitted with Langmuir-Hinshelwood pseudo first order equation with the rate constant of 0.0251 $\min ^{-1}$. Figure $8(d)$ represents the percentage reduction of $\mathrm{Cr}(\mathrm{VI})$ in the presence of $\mathrm{MoS}_{2}$ nanosheets and shows that $91.05 \% \mathrm{Cr}(\mathrm{VI}) \mathrm{re}$ duction was achieved in $75 \mathrm{~min}$.

3.2.4 Comparison of the effectiveness of the assynthesized $\mathrm{MoS}_{2}$ nanosheets with other published results

In order to ascertain the effectiveness of the as-synthesized $\mathrm{MoS}_{2}$ nanosheets over other photocatalysts in the degradation of $\mathrm{RhB}$ and $\mathrm{MB}$, and the reduction of $\mathrm{Cr}(\mathrm{VI})$, the photodegradation and photoreduction efficiencies of the $\mathrm{MoS}_{2}$ nanosheets have been matched with other published data (Table 1). Taking into consideration the concentrations of the catalysts and the pollutants used, and the duration
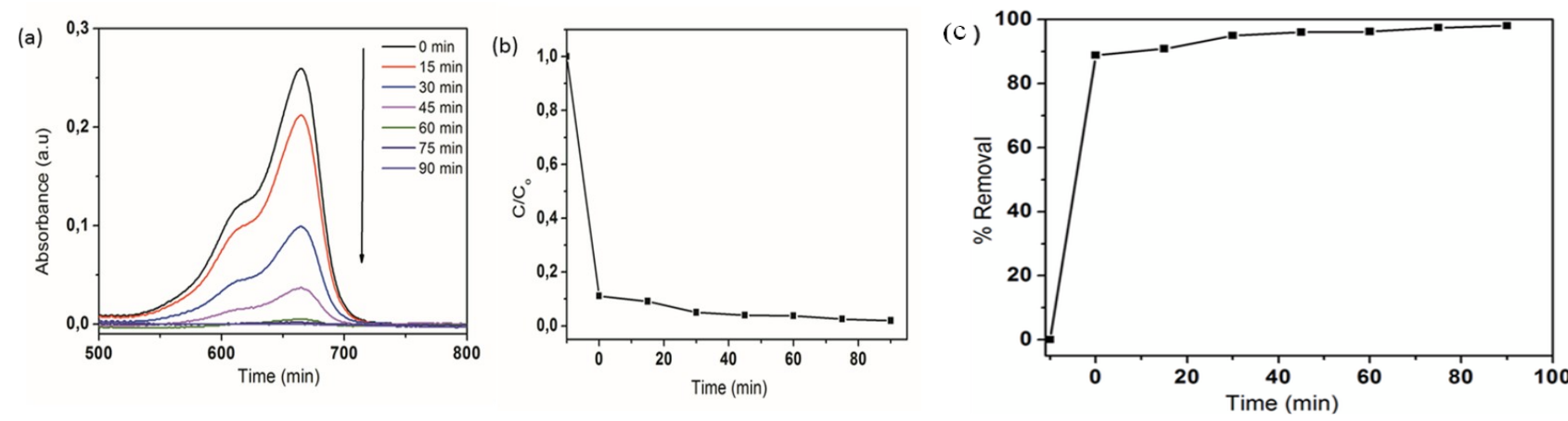

Figure 7. (a) UV-Vis spectroscopic changes of the MB aqueous solution in the presence $\mathrm{MoS}_{2}$ nanosheets, (b) Photocatalytic degradation rate of $\mathrm{MB}$ as a function of time and (c) Percent removal of $\mathrm{MB}$ in the presence of $\mathrm{MoS}_{2}$ nanosheets as a function of irradiation time 

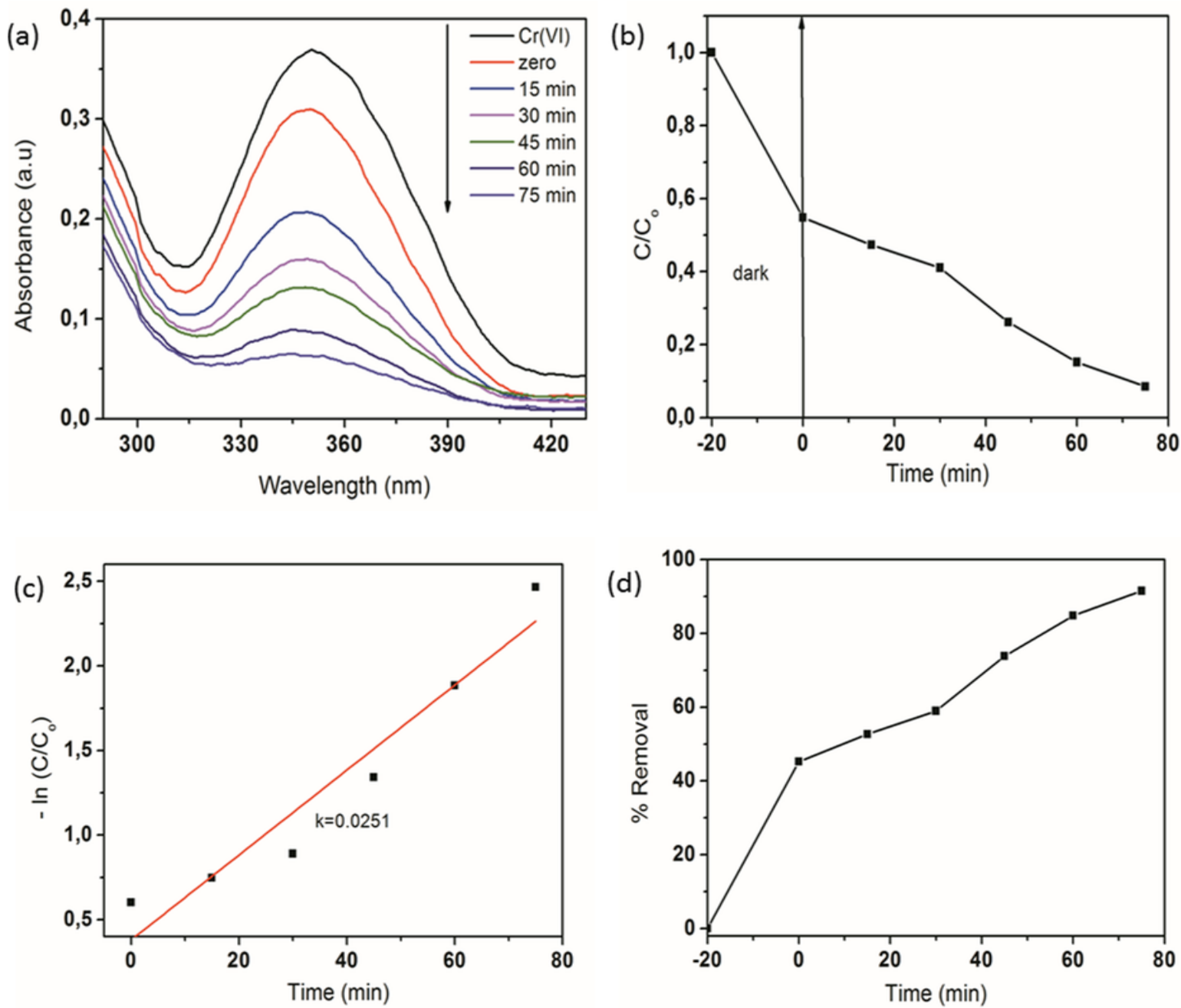

Figure 8. (a) UV-Vis absorption spectra of $\mathrm{Cr}(\mathrm{VI})$ reduction, (b) Photocatalytic reduction rate of $\mathrm{Cr}(\mathrm{VI})$, (c) Langmuir-Hinshelwood pseudo-first order kinetic plots or reduction of $\mathrm{Cr}(\mathrm{VI})$, and (d) Percent photoreduction of $\mathrm{Cr}(\mathrm{VI})$

Table 1. Comparison of the effectiveness of the as-synthesized MoS 2 nanosheets with other published resultson the photodegradation of $\mathrm{RhB}$ and $\mathrm{MB}$, and the photoreduction of $\mathrm{Cr}(\mathrm{VI})$ in wastewater using visible light

\begin{tabular}{|c|c|c|c|c|c|c|}
\hline Catayst name & $\begin{array}{c}\text { Pollutant } \\
\text { name }\end{array}$ & $\begin{array}{c}\text { Catalyst conc. } \\
(\mathrm{mg} / \mathrm{L})\end{array}$ & $\begin{array}{c}\text { Pollutant } \\
\text { conc. }(\mathrm{mg} / \mathrm{L})\end{array}$ & $\begin{array}{l}\text { Time } \\
(\mathrm{min})\end{array}$ & $\begin{array}{c}\text { Degradation } \\
(\%)\end{array}$ & Reference \\
\hline CuS/PVA & RhB & 20 & 50 & 60 & 80 & [27] \\
\hline $\mathrm{Au} / \mathrm{ZnO}$ & $\mathrm{RhB}$ & 100 & 20 & 60 & 87 & [28] \\
\hline $\mathrm{NaBiO}_{3}$ & $\mathrm{RhB}$ & 100 & 20 & 180 & 97 & [29] \\
\hline PEG-MoS 2 & $\mathrm{RhB}$ & 50 & 10 & 90 & 97 & [present work] \\
\hline Pure $\mathrm{MoS}_{2}$ & $\mathrm{RhB}$ & 50 & 10 & 90 & 72 & [present work] \\
\hline $\mathrm{TiO}_{2}$ & $\mathrm{MB}$ & 20 & 20 & 300 & 86 & [30] \\
\hline $\mathrm{ZnO}-\mathrm{SnO}_{2}$ & MB & 400 & 20 & 60 & 96 & [31] \\
\hline $\mathrm{TiO}_{2} / \mathrm{AC}$ & MB & 20 & 50 & 90 & 98 & [32] \\
\hline PEG-MoS ${ }_{2}$ & MB & 50 & 10 & 90 & 98 & [present work] \\
\hline Pure $\mathrm{MoS}_{2}$ & MB & 50 & 10 & 90 & 76 & [present work] \\
\hline $\mathrm{TiO}_{2}$ & $\mathrm{Cr}(\mathrm{VI})$ & 50 & 20 & 60 & 86 & [33] \\
\hline $\mathrm{NiFe}_{2} \mathrm{O}_{4}-\mathrm{SiO}_{2}$ & $\mathrm{Cr}(\mathrm{VI})$ & 200 & 10 & 300 & 97 & {$[34]$} \\
\hline $\mathrm{ZnO}$ & $\mathrm{Cr}(\mathrm{VI})$ & 1000 & 20 & 120 & 80 & [35] \\
\hline PEG-MoS ${ }_{2}$ & $\mathrm{Cr}(\mathrm{VI})$ & 50 & 10 & 90 & 91 & [present work] \\
\hline Pure $\mathrm{MoS}_{2}$ & $\mathrm{Cr}(\mathrm{VI})$ & 50 & 10 & 90 & 68 & [present work] \\
\hline
\end{tabular}


of the photocatalytic experiments, the degradation efficiencies of the as-synthesized $\mathrm{MoS}_{2}$ nanosheets evidenced that it is an effective catalyst as it showed much higher degradation efficiencies in the degradation of the two dyes and the reduction of $\mathrm{Cr}(\mathrm{VI})$ in comparison to the other photocatalysts used. In addition, the efficiency of the pure $\mathrm{MoS}_{2}\left(\mathrm{MoS}_{2}\right.$ without PEG 400) against the photodegradation of the two dyes (RhB and $\mathrm{MB}$ ) and the photoreduction of $\mathrm{Cr}$ (VI) was also compared with that of the pegylated $\mathrm{MoS}_{2}$ (Table 1). The results revealed that the pegylated $\mathrm{MoS}_{2}$ displayed higher efficiencies in all the processes compared to the pure $\mathrm{MoS}_{2}$. This observation indicates that the PEG 400, apart from acting as a capping agent, also enhanced the photodegradation efficiency of $\mathrm{MoS}_{2}$.

\subsubsection{Recyclability studies}

The ability of the photocatalyst to be regenerated and re-used has been tested. This property is an important requirement as it will assist in reducing the cost associated with using new photocatalysts during each cycle. The outcome of this analysis (Figure 9) show only a small variation within the catalyst's reusability over the five cycles in the degradation of the dyes (RhB and $\mathrm{MB}$ ) and the reduction of $\mathrm{Cr}(\mathrm{VI})$. The degradation efficiencies ranged from $97.3 \%$ to $91.7 \%$, and $98.05 \%$ to $92.2 \%$ within the $80 \mathrm{~min}$ irradiation time for $\mathrm{RhB}$ and $\mathrm{MB}$, respectively. Those of $\mathrm{Cr}(\mathrm{VI})$ reduction varied from $91.05 \%$ to $89.25 \%$. The results indicate that the $\mathrm{MoS}_{2}$ nanoparticle has

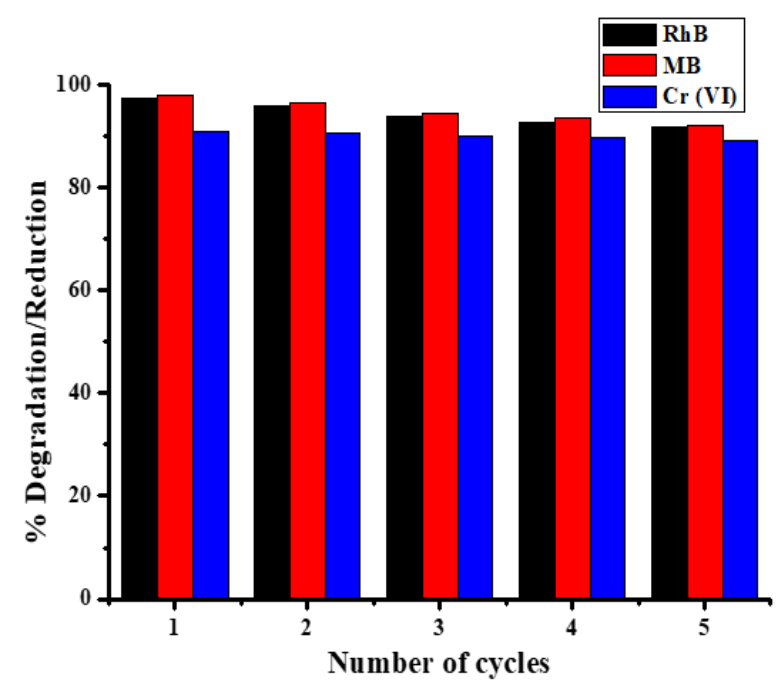

Figure 9. Recyclability study on $\mathrm{MoS}_{2}$ nanosheets for the degradation of $\mathrm{RhB}, \mathrm{MB}$, and reduction of $\mathrm{Cr}(\mathrm{VI})$ the potential to be effectively recycled and reused over a number cycles to proficiently degrade the dyes and reduce $\mathrm{Cr}(\mathrm{VI})$ to $\mathrm{Cr}$ (III) in aqueous medium.

\section{Conclusion}

In summary, the microspheres of $\mathrm{MoS}_{2}$ nanosheets were successfully synthesised by hydrothermal method. XRD reveals that the synthesised $\mathrm{MoS}_{2}$ nanosheets can be indexed to hexagonal structure of $\mathrm{MoS}_{2}$ (ICDD: 00-0371492). SEM, TEM, and HRTEM images show that the synthesised sample has spherical shapes made up of several thin sheets of $\mathrm{MoS}_{2}$ with the $d$-spacing of $0.682 \mathrm{~nm}$. The energy band gap was estimated to be $1.92 \mathrm{eV}$. The $\mathrm{RhB}$ and MB dyes experienced degradation efficiencies of $97.30 \%(\mathrm{RhB})$ and $98.05 \%(\mathrm{MB})$ by the catalyst in 75 min $90 \mathrm{~min}$, respectively in the presence of light. The results reveal that the synthesised $\mathrm{MoS}_{2}$ nanosheet is a good photocatalytic material for degradation of dyes in water. It was also observed that light plays a crucial role in the degradation of the dyes. The catalyst is also observed to be effective in the photocatalytic reduction of $\mathrm{Cr}(\mathrm{VI}) .91 .05 \%$ reduction of $\mathrm{Cr}(\mathrm{VI})$ to $\mathrm{Cr}$ (III) was achieved in $75 \mathrm{~min}$.

\section{Acknowledgements}

The authors wish to express their gratitude to the Faculty of Science, University of Johannesburg and the DST/MSc Nanoscience Programme for their financial support toward this research.

\section{References}

[1] Hoffmann, M.R., Scot, T.M., Choi, W., Bahnemann, D.W. (1995). Environmental Applications of Semiconductor Photocatalysis. Chemical Reviews, 95: 69-96.

[2] Pouretedal, H.R., Norozi, A., Keshavarz, M.H., Semnani, A. (2009). Nanoparticles of Zinc Sulfide Doped with Manganese, Nickel and Copper as Nanophotocatalyst in the Degradation of Organic Dyes. Journal of Hazardous Materials, 162: 674-681.

[3] Sohrabnezhad, S.H. (2011). Study of Catalytic Reduction and Photodegradation of Methylene Blue by Heterogeneous Catalyst. Spectrochimica Acta Part A: Molecular and Biomolecular Spectroscopy, 81: 228-235.

[4] Martínez, S.S., Uribe, E.V. (2012). Enhanced Sonochemical Degradation of Azure B Dye by the Electrofenton Process. Ultrasonics Sonochemistry, 19: 174-178. 
[5] Alluri, H.K., Ronda, S.R., Settalluri, V.S., Bondili, J.S., Suryanarayana, V., Venkateshwar, P. (2007). Biosorption: An EcoFriendly Alternative for Heavy Metal Removal. African Journal of Biotechnology, 6: 2924-2931.

[6] Robinson, T., McMullan, G., Marchant, R., Nigam, P. (2001). Remediation of Dyes in Textile Effluent: A Critical Review on Current Treatment Technologies with a Proposed Alternative. Bioresource Technology, 77: 247-255.

[7] Slokar, Y.M., Le Marechal, A.M. (1998). Methods of Decoloration of Textile Wastewaters. Dyes and Pigments, 37: 335-356.

[8] Víctor-Ortega, M.D., Ochando-Pulido, J.M., Hodaifa, G., Martinez-Ferez, A. (2014). Final Purification of Synthetic Olive Oil Mill Wastewater Treated by Chemical Oxidation Using Ion Exchange: Study of Operating Parameters. Chemical Engineering and Processing: Process Intensification, 85: 241-247.

[9] Hu, H., Yang, M., Dang, J. (2005). Treatment of Strong Acid Dye Wastewater by Solvent Extraction. Separation and Purification Technology, 42: 129-136.

[10] Yagub, M.T., Sen, T.K., Afroze, S., Ang, H.M. (2014). Dye and Its Removal from Aqueous Solution by Adsorption: A Review. Advances in Colloid and Interface Science, 209: 172-184.

[11] Xu, L., Sun, Y., Du, L., Zhang, J. (2014). Removal of Tetracycline Hydrochloride from Wastewater by Nanofiltration Enhanced by Electro-Catalytic Oxidation. Desalination, 352: 58-65.

[12] Zhou, S., Watanabe, H., Wei, C., Wang, D., Zhou, J., Tatarazako, N., Masunaga, S., Zhang, Y. (2015). Reduction in Toxicity of Coking Wastewater to Aquatic Organisms by Vertical Tubular Biological Reactor. Ecotoxicology and Environmental Safety, 115: 217-222.

[13] Kudo, A., Miseki, Y. (2009). Heterogeneous Photocatalyst Materials for Water Splitting. Chemical Society Reviews, 38: 253-278.

[14] Chong, M.N., Jin, B., Chow, C.W., Saint, C. (2010). Recent Developments in Photocatalytic Water Treatment Technology: A Review. Water Research, 44: 2997-3027.

[15] Kramer, T.J., Babu, S.S., Saeki, A., Seki, S., Aimi, J., Nakanishi, T. (2012). CdSe Nanocrystal/C 60-Liquid Composite Material with Enhanced Photoelectrochemical Performance. Journal of Materials Chemistry, 22: 22370-22373.

[16] Sun, X.F., Liu, B., Jing, Z., Wang, H. (2015). Preparation and Adsorption Property of Xylan/Poly (Acrylic Acid) Magnetic Nanocomposite Hydrogel Adsorbent. Carbohydrate Polymers, 118: 16-23.
[17] Li, Y., Du, Q., Liu, T., Peng, X., Wang, J., Sun, J., Wang, Y., Wu, S., Wang, Z., Xia, Y., Xia, L. (2013). Comparative Study of Methylene Blue Dye Adsorption onto Activated Carbon, Graphene Oxide, and Carbon Nanotubes. Chemical Engineering Research and Design, 91: 361-368.

[18] Chitkara, M., Singh, K., Sandhu, I.S., Bhatti, H.S. (2011). Photo-Catalytic Activity of $\mathrm{Zn}_{1}$. ${ }_{x} \mathrm{Mn}_{\mathrm{x}} \mathrm{S}$ Nanocrystals Synthesised by Wet Chemical Technique. Nanoscale Research Letters, 6: 1-1

[19] Datta, A., Priyam, A., Bhattacharyya, S.N., Mukherjea, K.K., Saha, A. (2008). Temperature Tunability of Size in CdS Nanoparticles and Size Dependent Photocatalytic Degradation of Nitroaromatics. Journal of Colloid and Interface Science, 322: 128-135.

[20] Wu, C., Huang, X., Xie, L., Wu, X., Yu, J., Jiang, P. (2011). Morphology-Controllable Graphene- $\mathrm{TiO}_{2}$ Nanorod Hybrid Nanostructures for Polymer Composites with High Dielectric Performance. Journal of Materials Chemistry, 21: 17729-17736.

[21] Chen, H., Nanayakkara, C.E., Grassian, V.H. (2012). Titanium Dioxide Photocatalysis in Atmospheric Chemistry. Chemical Reviews, 112: 5919-5948.

[22] Liu, G., Zhao, Y., Sun, C., Li, F., Lu, G.Q., Cheng, H.M. (2008). Synergistic Effects of B/N Doping on the Visible-Light Photocatalytic Activity of Mesoporous $\mathrm{TiO}_{2}$. Angewandte Chemie International Edition, 47: 4516-4520.

[23] Asahi, R., Morikawa, T., Ohwaki, T., Aoki, K., Taga, Y. (2001). Visible-Light Photocatalysis in Nitrogen-Doped Titanium Oxides, Science, 293: 269-271.

[24] Macphee, D.E., Rosenberg, D., Skellern, M.G., Wells, R.P., Duffy, J.A., Killham, K.S. (2011). A Tungsten Oxide-Based Photoelectrocatalyst for Degradation of Environmental Contaminants, Journal of Solid State Electrochemistry, 15: 99-103.

[25] Lee, H.S., Min, S.W., Chang, Y.G., Park, M.K., Nam, T., Kim, H., Kim, J.H., Ryu, S., Im, S. (2012). $\mathrm{MoS}_{2}$ Nanosheets Phototransistors with Thickness-Modulated Optical Energy Gap. Nano Letters, 12: 3695-3700.

[26] Wang, Q.H., Kalantar-Zadeh, K., Kis, A., Coleman, J.N., Strano, M.S. (2012). Electronics and Optoelectronics of Two-Dimensional Transition Metal Dichalcogenides. Nature Nanotechnology, 7: 699-712.

[27] Al-Kahtani, A.A. (2016). Photocatalytic Degradation of Rhodamine B Dye in Wastewater Using Gelatin/CuS/PVA Nanocomposites under Solar Light Irradiation. Journal of Biomaterials and Nanobiotechnology, 8: 66-68. 
[28] Alshammari, A., Bagabas, A., Assulami, M (2014). Photodegradation of Rhodamine B over Semiconductor Supported Gold Nanoparticles: The Effect of Semiconductor Support Identity. Arabian Journal of Chemistry. doi.org/10.1016/j.arabjc.2014.11.013

[29] Yu, K., Yang, S., He, H., Sun, C., Gu, C., Ju, Y. (2009). Visible Light-Driven Photocatalytic Degradation of Rhodamine $\mathrm{B}$ over $\mathrm{NaBiO}_{3}$ : Pathways and Mechanism. The Journal of Physical Chemistry A, 113: 10024-10032.

[30] Dariani, R. S., Esmaeili, A., Mortezaali, A., Dehghanpour, S. (2016). Photocatalytic Reaction and Degradation of Methylene Blue on $\mathrm{TiO}_{2}$ Nano-Sized Particles. OptikInternational Journal for Light and Electron Optics, 127: 7143-7154.

[31] Lin, J., Luo, Z., Liu, J., Li, P. (2018). Photocatalytic Degradation of Methylene Blue in Aqueous Solution by Using $\mathrm{ZnO}-\mathrm{SnO}_{2}$ Nanocomposites. Materials Science in Semiconductor Processing, 87: 24-31.

[32] Ramli, C., Amali, Z., Asim, N., Isahak, W.N., Emdadi, Z., Ahmad-Ludin, N., Sopian, K. (2014). Photocatalytic Degradation of Methylene Blue under UV Light Irradiation on Prepared Carbonaceous. The Scientific World Journal, 2014: 1-8 (Article ID 415136, (doi:10.1155/2014/415136)
[33] Wu, Q., Zhao, J., Qin, G., Wang, C., Tong, X., Xue, S. (2013). Photocatalytic Reduction of $\mathrm{Cr}(\mathrm{VI})$ with $\mathrm{TiO}_{2}$ Film under Visible Light. Applied Catalysis B: Environmental, 142: 142-148.

[34] Ojemaye, M.O., Okoh, O.O., Okoh, A.I. (2017). Performance of $\mathrm{NiFe}_{2} \mathrm{O}_{4}-\mathrm{SiO}_{2}-\mathrm{TiO}_{2}$ Magnetic Photocatalyst for the Effective Photocatalytic Reduction of $\mathrm{Cr}(\mathrm{VI})$ in Aqueous Solutions. Journal of Nanomaterials, 2017: 1-11 (Article ID 5264910, doi:10.1155/2017/ 5264910)

[35] Shirzad, S.M., Samadi, M.T., Yang, J.K., Lee, S.M. (2011). Photocatalytic Reduction of $\mathrm{Cr}$ (VI) and Ni (II) in Aqueous Solution by Synthesized Nanoparticle $\mathrm{ZnO}$ under Ultraviolet Light Irradiation: A Kinetic Study. Environmental Technology, 32: 15731579 . 